\title{
Prognostic factors for local control of stage I non-small cell lung cancer in stereotactic radiotherapy: a retrospective analysis
}

Yuko Shirata ${ }^{1,4^{*}}$, Keiichi Jingu', Masashi Koto ${ }^{2}$, Masaki Kubozono ${ }^{1}, K^{1}$ Ken Takeda ${ }^{3}$, Toshiyuki Sugawara', Noriyuki Kadoya' and Haruo Matsushita'

\begin{abstract}
Background: The purpose of this study is to investigate the prognostic factors of stereotactic radiotherapy for stage I NSCLC to improve outcomes.

Methods: Stage I non-small cell lung cancer patients who were treated with stereotactic radiotherapy between 2005 and 2009 at our hospital were enrolled in this study. The primary endpoint was local control rate. Survival estimates were calculated from the completion date of radiotherapy using the Kaplan-Meier method. The prognostic factors including patients' characteristics and dose-volume histogram parameters were evaluated using Cox's proportional hazard regression model.

Results: Eighty patients (81 lesions) treated with 3 dose levels, 48 Gy/4 fractions, 60 Gy/8 fractions and 60 Gy/15 fractions, were enrolled in this study. Median follow-up was 30.4 months (range, $0.3-78.5$ months). A Cox regression model showed $T$ factor $(p=0.013)$, biological effective dose calculated from prescribed dose $\left(B_{E D} D_{10}\right)$ ( $p=0.048)$, and minimum dose for PTV $(p=0.013)$ to be prognostic factors for local control. Three-year overall survival rate and local control rate were 89.9\% (T1: 86.8\%, T2: 100\%) and 89.0\% (T1: 97.9\%; T2: 64.8\%), respectively. When the 3-year local control rates were examined by prescribed doses, they were 100\% for the dose per fraction of $48 \mathrm{~Gy} / 4$ fractions (105.6 Gy BED 10 ), 82.1\% for 60 Gy/8 fractions (105 Gy BED 10 ), and 57.1\% for 60 Gy/15 fractions (84 Gy $\mathrm{BED}_{10}$ ). The median value of the minimum dose for PTV (\%) was $89.88(\%)$, and the 3-year local control rates were $100 \%$ in those with the minimum dose for PTV (\%) $\geq 89.88 \%$ and $79.2 \%$ in those with the minimum dose for PTV (\%) < 89.88\%.
\end{abstract}

Conclusions: Our results suggest that $\mathrm{T}$ factor, $\mathrm{BED}_{10}$, and minimum dose for PTV influence the local control rate. Local control rate can be improved by securing the minimum dose for PTV.

Keywords: Stereotactic radiotherapy, SBRT, Non-small cell lung cancer, NSCLC, Prognostic factor, Minimum dose, PTV margin

\section{Background}

In stereotactic radiotherapy for lung tumors, the dose at the lesion has been successfully increased through advancement of irradiation devices, improvement of set-up accuracy, introduction of image-guidance technology and measures for respiratory tumor movement $[1,2]$

\footnotetext{
* Correspondence: guldoktu@gmail.com

'Department of Radiation Oncology, Tohoku University School of Medicine, Sendai, Japan

${ }^{4}$ Department of Radiation Oncology, Tohoku University School of Medicine, 1-1 Seiryo-chou, Aoba-ku, Sendai 980-8574, Japan

Full list of author information is available at the end of the article
}

while ensuring a high level of safety. For stage I nonsmall cell lung cancer (NSCLC), in particular, some reports suggest that short-term outcomes of stereotactic radiotherapy are comparable to those of surgeries [3,4]. Furthermore, patients diagnosed with lung cancer in the early stage have increased recently due to the use of computed tomography (CT) scans and educational campaigns for cancer screening $[5,6]$.

However, lung cancer is still the main cause of cancer death worldwide, and local recurrence after stereotactic radiotherapy is not rare [3,7-9]. The purpose of this

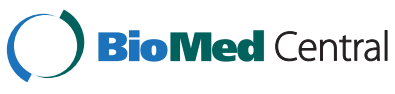


study is to investigate the prognostic factors of stereotactic radiotherapy for stage I NSCLC to improve outcomes.

\section{Methods}

\section{Patient eligibility}

We reviewed data for all of the 80 patients (81 lesions) with stage I NSCLC who had undergone stereotactic radiotherapy in our hospital between March 2005 and July 2009. All patients included in this study had histological or cytological diagnosis of NSCLC and were staged as Union Internationale Contre le Cancer (UICC)-6 stage IA or IB by the use of CT. If available, 18 F-fluorodeoxyglucose positron emission tomography (FDG-PET) was used for staging. They either had a medical contraindication to surgery or refused surgery. Patient eligibility was not restricted on the basis of tumor location, unless a part of the esophagus, heart, main bronchi, hilus, spinal cord, or skin would be exposed to high-dose radiation. If the treatment plan included these organs in the high-dose areas, the patients were treated with conventional radiotherapy or modified stereotactic radiotherapy with a moderate irradiation dose; those patients were excluded from this study.

The primary endpoint was local recurrence. The secondary endpoint was overall survival. Patients' background factors, various clinical parameters, and clinical course after irradiation were surveyed using information sources including medical records, data saved at the practice support terminal of our hospital, case follow-up cards of our department, and irradiation records. Local recurrence was defined as local progression that was 1.5 times or more the dimensions of original tumor [10]. Tumors were observed on CT and/or 18 F-fluorodeoxyglucose positron emission tomography (FDG-PET) in order to assess the primary tumor's stage and the presence or absence of recurrence. If FDG-PET was available, the maximum standardized uptake value (SUVmax) greater than 5.0 was considered as recurrence [11]. The physicians and radiation oncologists finally decided to be local recurrence.

The study protocol was approved by the Ethical Committee of our institution, and informed consent was obtained from all patients.

\section{Radiotherapy}

Treatment factor is summarized in Table 1. To determine the extent of tumor movement due to respiration and establish an individual internal margin, all patients were placed in a simulator for fluoroscopic examination just prior to CT scanning for treatment planning in the exact same position. Serial CT scans were performed at intervals of $2.5 \mathrm{~mm}$. A CT scan with an acquisition time
Table 1 Treatment factor

\begin{tabular}{|c|c|}
\hline \multirow{2}{*}{$\begin{array}{l}\text { Computed } \\
\text { tomography }\end{array}$} & - Long time scan \\
\hline & - $2.5 \mathrm{~mm}$ slice \\
\hline \multirow{3}{*}{$\begin{array}{l}\text { Radiotherapy planning } \\
\text { system }\end{array}$} & - Eclipse \\
\hline & -Algorithm: Pencil beam convolution \\
\hline & $\begin{array}{l}\text { - Heterogeneity correction: Modified Batho } \\
\text { Power Law }\end{array}$ \\
\hline \multirow[t]{2}{*}{ Targeting } & $\begin{array}{l}\text { - Observation of the tumor by fluoroscopy } \\
\text { in advance }\end{array}$ \\
\hline & $\begin{array}{l}-\mathrm{GTV}+0-5 \mathrm{~mm}=\mathrm{CTV}, \mathrm{CTV}+5-10 \mathrm{~mm}= \\
\text { PTV }\end{array}$ \\
\hline Irradiation field & $\begin{array}{l}\text { - non-coplanar multi-dynamic arcs and/or } \\
\text { multi-static beams }\end{array}$ \\
\hline \multirow[t]{3}{*}{ Prescription } & $\begin{array}{l}-48 \text { Gy/4 fractions, } 60 \text { Gy/8 fractions, } \\
60 \text { Gy/15 fractions }\end{array}$ \\
\hline & - Prescription for the iso-center \\
\hline & - 6 MV - X ray \\
\hline $\begin{array}{l}\text { Radiation therapy } \\
\text { equipment }\end{array}$ & - Clinac 23EX \\
\hline
\end{tabular}

of 4 seconds that included internal motion was performed to define the internal margin accurately.

Patients were immobilized in the supine position with an individually fashioned half-body vacuum cast. Both the upper extremities were immobilized in the raised position unless the tumor was located at the apex of the lung, in which case both the upper extremities were immobilized beside the body.

Gross tumor volume (GTV) was defined as the visible extent of the tumor on the CT image at the lung window. Clinical target volume (CTV) was defined as GTV plus $0-5 \mathrm{~mm}$ margin for microscopic invasion. Internal target volume (ITV) was set equal to CTV because CT scanning was performed with an acquisition time of 4 seconds, and we consider that long-time (4 seconds) scan CT depicted virtually the entire tumor trajectory [12]. Planning target volume (PTV) was determined by allowing for a set-up margin of $5-10 \mathrm{~mm}$ beyond the ITV.

Treatment planning was performed with non-coplanar multi-dynamic arcs and/or multi-static beams by using a three-dimensional radiotherapy treatment planning system (Eclipse, Varian Medical Systems, Palo Alto, CA). The algorithm to calculate the dose was pencil beam convolution (PBC). Modified Batho Power law correction was used as the tissue heterogeneity correction algorithm. The target reference point was defined as the center of the PTV, and the dose was prescribed for its point. PTV was encompassed by the minimum $90 \%$ dose line of the reference point dose as possible. X-rays of 6 MV were used in all treatments.

The treatment took place using Clinac 23EX, Varian Medical Systems, Palo Alto, CA. In each irradiation, the 
position of the tumor was confirmed with fluoroscopy, and set-up and/or inter-fractional errors were corrected.

The patients were treated with a radiation schedule of 12 Gy /fraction $\times 4$ fractions (48 Gy/4 fractions), $7.5 \mathrm{~Gy} /$ fraction $\times 8$ fractions (60 Gy/8 fractions), and $4 \mathrm{~Gy} /$ fraction $\times 15$ fractions (60 Gy/15 fractions). When the tumor was close to a risk organ, $7.5 \mathrm{~Gy} /$ fraction $\times 8$ fractions or $4 \mathrm{~Gy} /$ fraction $\times 15$ fractions was used to reduce the risk of serious toxicity due to set-up error or internal motion.

\section{Follow-up}

The first examination, including a clinical examination and CT scanning, was performed 4-6 weeks after treatment to assess the pulmonary reaction. Thereafter, the patients underwent follow-up examinations every 3-6 months for 2 years following treatment. After 2 years, follow-up examinations were performed every 6 months.

\section{Statistical analysis}

The prognostic factors for local control, including age, sex, T factor, histology, planning target volume (PTV), minimum dose for PTV (\%), biological effective dose (BED) calculated from prescribed dose $\left(\mathrm{BED}_{10}\right)$, and biological effective dose calculated from minimum dose (BEDmin), were investigated by stepwise Cox's proportional hazard regression model for multivariate analysis. Hazard ratio for continuous data was evaluated with Wald $x^{2}$ test statistics. Sex and $T$ factor were considered as categorical data. To investigate for the presence of multicollinearity, correlation coefficients were calculated for all variables. The hazard ratio was observed graphically to check its constancy.

$\mathrm{BED}_{10}$ and BEDmin were calculated using the linear quadratic formula in order to compare the effects of treatments with different fraction sizes and total doses. BED was given by: $\mathrm{BED}=\mathrm{nd}[1+\mathrm{d} /(\alpha / \beta)]$, where $n$ is the number of fractions, $\mathrm{d}$ is the dose/fraction, and $\alpha / \beta$ ratio is $10 \mathrm{~Gy}$. BED was not corrected with values for overall radiation time or tumor doubling time.

Overall survival, cause specific, and local control rates were calculated using the Kaplan-Meier method and statistical differences were evaluated by the log-rank test. When a continuous data was used as a variable for the Kaplan-Meier method, the data was divided by the median value into two groups. Statistical significance was defined as a value of $\mathrm{p}<0.05$ in the present study. All analyses were performed using the SPSS 17.0 software package (SPSS Inc, Chicago, IL).

\section{Results}

Patients

All eighty patients were enrolled in the present study. The patients' characteristics are shown in Table 2. There were
64 men (64 lesions) and 16 women (17 lesions) with ages ranging from 54 to 90 years (median, 70 years). All of the patients completed the treatment without acute adverse effects. The observation periods from the time of completion of stereotactic radiotherapy ranged from 0.3 to 78.5 months with a median of 30.4 months. The patients' histologies were adenocarcinoma (33 patients), squamous cell carcinoma (22 patients), large cell carcinoma (5 patients), and unclassified NSCLC (20 patients). Sixty-three tumors were T1 (Stage IA) masses and 18 tumors were T2 (Stage IB). Performance status (PS) of the patient was $0-2$.

Fifteen $(18.8 \%)$ of the 80 patients showed evidence of recurrence. Local, regional (nodal) and distant recurrences were observed in 6 patients $(7.5 \%), 3$ patients (3.8\%) and 12 patients (15\%), respectively. Time to local failure varied between 12.2 and 33.7 months (median, 18.1 months).

Four patients died of NSCLC treated with stereotactic radiotherapy and 6 patients died of intercurrent causes. The 4 patients who died of NSCLC treated with stereotactic radiotherapy included 1 patient with local disease and regional lymph node metastasis and 3 patients with distant metastases. Intercurrent causes were colorectal cancer, aspiration pneumonia, advanced esophageal cancer, renal failure, chronic obstructive pulmonary disease (COPD), and multiple liver metastases considered to be from another site of NSCLC.

\section{Radiotherapy}

We treated 45 patients with a radiation schedule of $12 \mathrm{~Gy} /$ fraction $\times 4$ fractions, 29 patients with a schedule of $7.5 \mathrm{~Gy} /$ fraction $\times 8$ fractions and 7 patients with a schedule of $4 \mathrm{~Gy} /$ fraction $\times 15$ fractions. We treated the tumor in consecutive weekdays.

\section{Statistical analysis}

The results of multivariate analysis showed significant differences in $\mathrm{T}$ factor, $\mathrm{BED}_{10}$ and minimum dose for PTV (\%). The hazard ratios were 0.027, 0.383 and 0.731, respectively (Table 3 ). Namely, the hazard ratio of T1 was 0.027 on defining the hazard ratio of T2 as 1.0. In

\section{Table 2 Patient backgrounds}

\begin{tabular}{ll}
\hline Age & median, 77 years (range: $54-90$ years) \\
Sex & Male: 64 lesions, 64 patients \\
& Female: 17 lesions, 16 patients \\
Histopathology & Adenocarcinoma: 33 patients \\
& Squamous cell carcinoma: 22 patients \\
& Large cell carcinoma: 5 patients \\
& Unclassified: 20 patients \\
Stage & T1 (Stage IA): 63 lesions \\
& T2 (Stage IB): 18 lesions
\end{tabular}


minimum dose for PTV, the hazard ratio for each $1 \%$ increase was 0.731 for local recurrence. In $\mathrm{BED}_{10}$ (Gy), the hazard ratio for each 10 Gy increase was 0.383 . Wald $\chi^{2}$ test indicated that minimum dose for PTV (\%) was the strongest prognostic factor among these variables. There was no multicollinearity problem in this study. We observed constant hazard ratio graphically.

The 3-year local control rates were $89.0 \%$ (95\% confidence interval [CI], $80.4 \%-97.6 \%)$ in all patients, $97.9 \%$ (95\% CI, 93.8\% - 102.0\%) in those with T1 tumors and $64.8 \%(95 \% \mathrm{CI}, 38.9 \%-90.7 \%)$ in those with T2 tumors. The log-rank test showed a significant difference between these two groups ( $p=0.001$, Figure 1$)$. When the 3 -year local control rates were examined by prescribed doses, they were $100 \%$ for the dose per fraction of 12 Gy /fraction $\times 4$ fractions (105.6 Gy BED 10 ), 82.1\% (95\% CI, $63.1 \%-101.1 \%)$ for 7.5 Gy/fraction $\times 8$ fractions (105 Gy $\mathrm{BED}_{10}$ ), and $57.1 \%$ (95\% CI, 20.4\% - 93.8\%) for 4. Gy/fraction $\times 15$ fractions ( 84 Gy $\mathrm{BED}_{10}$ ) with a significant difference $(p=0.001$, Figure 2$)$. The median value of the minimum dose for PTV (\%) was 89.88 (\%), and the 3-year local control rates were $100 \%$ in those with the minimum dose for PTV (\%) $\geq 89.88 \%$ and $79.2 \%$ (95\% CI, 63.9\% - 94.5\%) in those with the minimum dose for PTV $(\%)<89.88 \%$. The log-rank test showed a significant difference between these two groups $(p=$ 0.016, Figure 3).

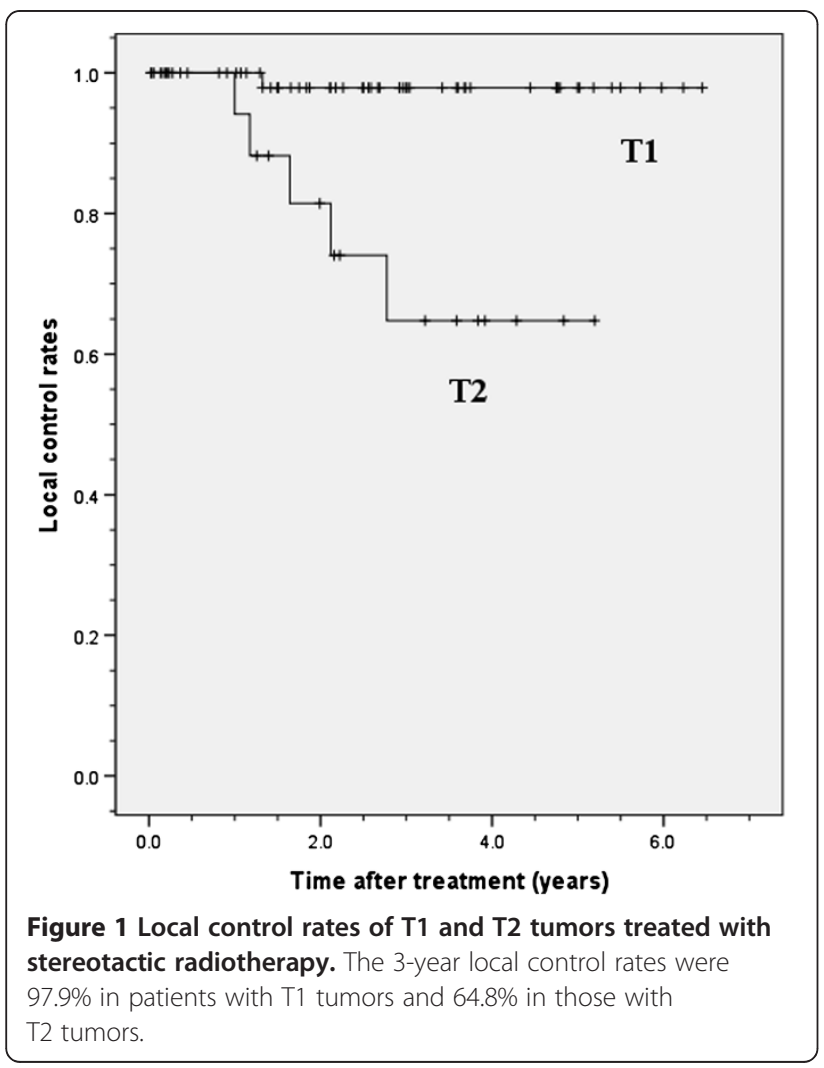

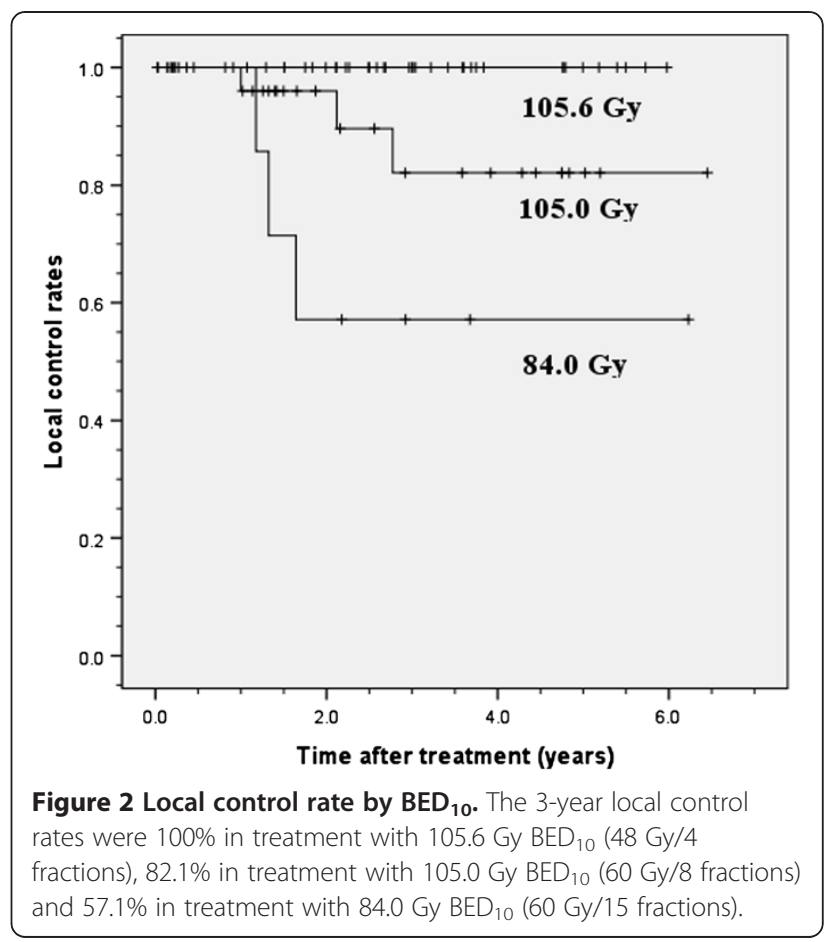

The 3-year overall survival rate for all patients was 89.9\% (95\% CI, $81.9 \%$ - 97.9\%). The 3-year overall survival rates were $86.8 \%(95 \% \mathrm{CI}, 76.6 \%$ - 97.0\%) in patients with $\mathrm{T} 1$ tumors and $100 \%$ in those with $\mathrm{T} 2$ tumors. No significant difference was observed between these two groups (log-rank test, $p=0.29$ ). The 3 -year cause specific survival rate was $97.0 \%$ (95\% confidence interval, 92.9\% - 101.1\%).

\section{Discussion}

Multivariate analysis indicated that $\mathrm{T}$ factor, $\mathrm{BED}_{10}$, and minimum dose for PTV (\%) influence local control. Among these, BED and minimum dose for PTV (\%) can be changed by artificial intervention. $\mathrm{BED}_{10}$ would depend on the dose per fraction and total dose at a dose prescription, and they are generally determined by patient's factor including tumor location, size, and patient's general condition. The minimum dose for PTV (\%) is decided by the radiotherapy plan including a targeting, margin factor, respiratory gating technique, and tumor tracking method. The results of this study suggest that the local control rate after stereotactic radiotherapy for stage I NSCLC can be improved by securing the minimum dose for PTV (\%) when radiation oncologists produce a radiotherapy plan.

\section{Prognostic factors}

Onimaru et al. analyzed 41 patients with stage I NSCLC treated by stereotactic radiotherapy and reported that $\mathrm{T}$ factor and prescribed dose were significant factors for 
Table 3 Multivariate analysis with variables selected by stepwise method

\begin{tabular}{lllll}
\hline $\begin{array}{l}\text { Variable } \\
\text { [Reference] }\end{array}$ & $\begin{array}{l}\text { Hazard } \\
\text { ratio }\end{array}$ & $\mathbf{X}^{\mathbf{2}}$ & $\begin{array}{l}\text { 95\% confidence } \\
\text { interval of hazard } \\
\text { ratio }\end{array}$ & $\begin{array}{l}\text { Significance } \\
\text { probability }(\boldsymbol{p})\end{array}$ \\
\hline $\begin{array}{l}\text { T factor [Group: } \\
\text { T2] }\end{array}$ & 0.027 & 6.142 & $0.002-0.470$ & 0.013 \\
$\begin{array}{l}\text { Minimum dose } \\
\text { for PTV (\%) }\end{array}$ & 0.731 & 6.203 & $0.571-0.935$ & 0.013 \\
$\begin{array}{l}\text { [Unit: 1\%] } \\
\text { BED }\end{array}$ (Gy) [Unit: & 0.383 & 3.898 & $0.148-0.993$ & 0.048 \\
\hline 10 Gy] & & & & \\
\hline
\end{tabular}

local control in multivariate analysis. The margin status was not a significant factor in spite of the fact that it was used as a variable [2]. Probable reasons were the heterogeneity of the patients and the confounding factors analyzed in their study. Patients in whom a narrow margin was used included 7 patients with a T1 tumor and 3 patients with a T2 tumor.

In contrast, another study showed that tumor diameter and gender were the most significant factors affecting outcomes after stereotactic radiotherapy in recursive partitioning analysis; for comparison, tumor diameter was the only significant factor for local progression in a Cox proportional hazards model, and no margin-related variable was used [13]. This was possibly because of the female pathological inclination to have adenocarcinoma. Some reports suggest that adenocarcinoma patients with stage I NSCLC tend to have a better survival [14]. Since

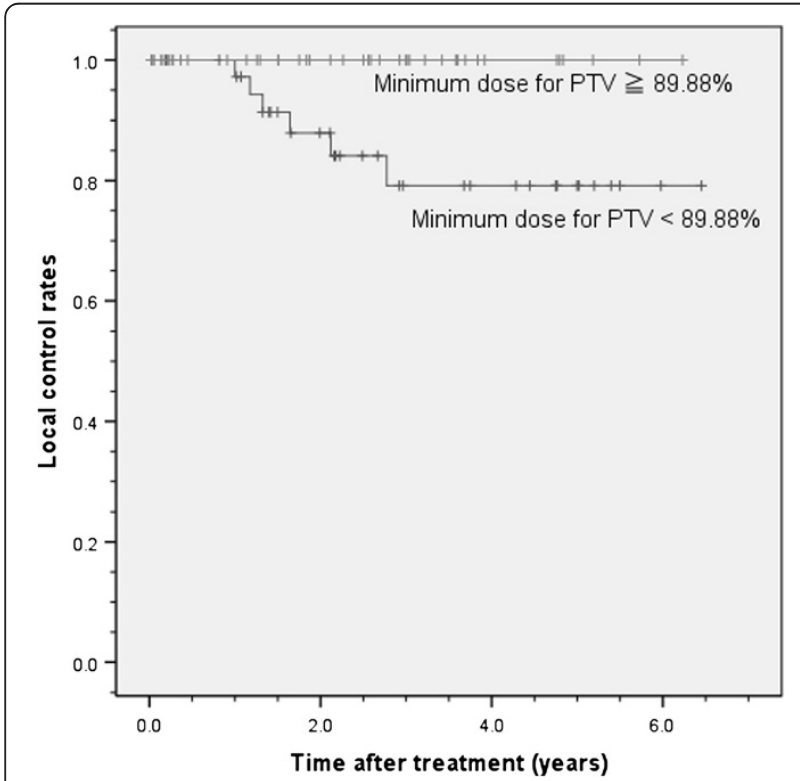

Figure 3 Local control rate by the minimum dose for PTV (\%). The 3-year local control rates were $100 \%$ in those with the minimum dose for PTV (\%) $\geq 89.88 \%$ and $79.2 \%(95 \% \mathrm{Cl}$,

$63.9 \%-94.5 \%)$ in those with the minimum dose for PTV $(\%)<89.88 \%$. $89.88 \%$ is the median value of the minimum dose for PTV (\%). only $16(20 \%)$ of the patients in our study were females, it was difficult to determine whether female gender is a significant prognostic factor.

Small sample size and retrospective protocol limit further interpretation of such findings, while tumor size is regarded as an independent prognostic factor for stage I NSCLC patients in general [14]. Multi-institutional phase II trials are currently be conducted by the Radiation Therapy Oncology Group (Protocol 0236) and Japan Clinical Oncology Group (Protocol 0403). Results of detailed statistical analysis of data obtained in these trials are awaited to clarify various issues including issues discussed below.

\section{T factor}

This study also showed that $\mathrm{T}$ factor is the most significant factor affecting local control after stereotactic radiotherapy for stage I NSCLC. Several studies have shown that local recurrence is more frequent for larger GTVs $[2,3,15]$ and that tumor size is an independent prognostic factor for stage I NSCLC patients [14]. A larger tumor tends to have hypoxic cells and cancer stem cells, which are more aggressive and resistant to radiotherapy [16], and a larger tumor would therefore have more time to metastasize than a smaller one. Cancer stem cells are recognized as a source of local or distant relapse [17]. A tendency to decrease the dose in the tumor periphery would also exacerbate the situation. In this study, the minimum dose for PTV (\%) tended to be slightly lower for larger PTV (Figure 4). In addition, $66.7 \%$ of the patients with a T2 tumor and $38 \%$ of the patients with a T1 tumor were prescribed $7.5 \mathrm{~Gy} / \mathrm{frac}-$ tion $\times 8$ fractions $\left(105\right.$ Gy $\left.\mathrm{BED}_{10}\right)$ or 4 Gy/fraction $\times 15$ fractions ( 84 Gy $\mathrm{BED}_{10}$ ). Hence, T2 tumors tended to be treated with longer overall radiation time than did $\mathrm{T} 1$ tumors.

Figure 1 shows that there was a significant difference between local control rates in patients with T1 and T2 tumor. There was a decline in local recurrence and a plateau at about three years with no local recurrence after that period, although late recurrences at 5 years or more have been reported [18]. Local control in our hospital was almost same as several reports (Table 4). The 3-year survival rate in our study was better than that in other studies. It might be due to good collaboration with other departments and/or other hospitals, that is, not only death of primary tumor but also death of other causes more fewer than previously, because three-year cause specific survival rate was 97.0 (\%), and it was almost same as some reports [19]. Relatively good survival rate could also imply that additional treatments after relapse including chemotherapy, re-irradiation, and best supportive care can influence survival. In addition, operability and PS may have affected the results of survival. 


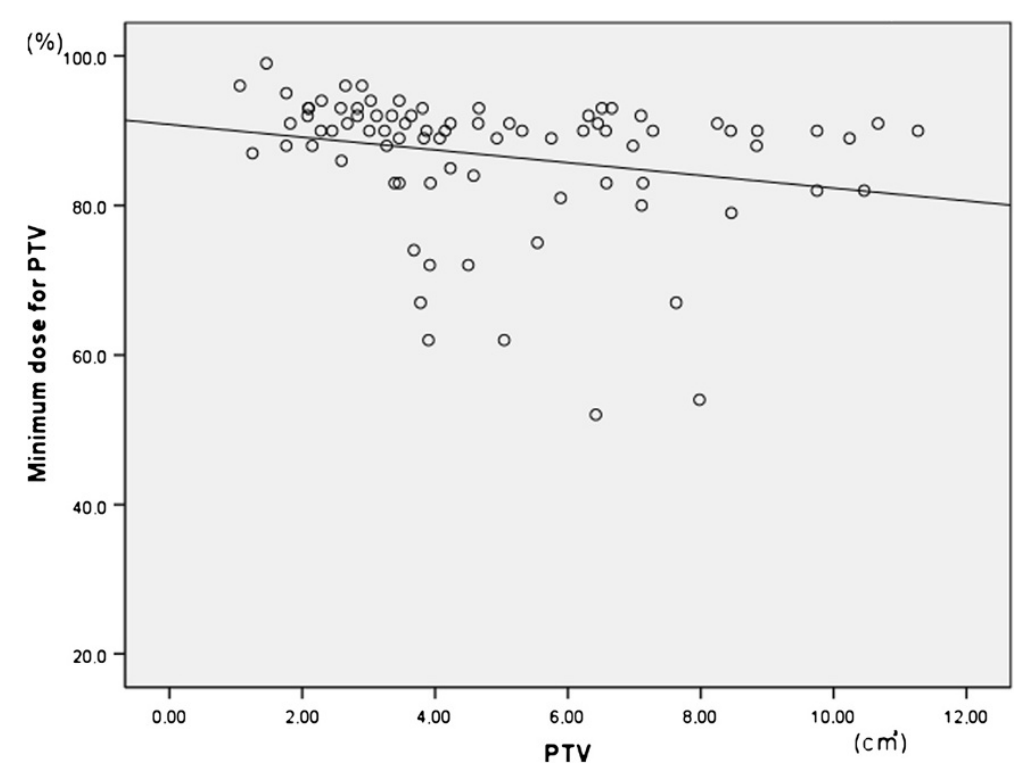

Figure 4 Correlation of Minimum dose for PTV (\%) and PTV. Minimum dose for PTV (\%) tended to be slightly lower for larger PTV.

$\mathrm{BED}_{10}$

When two types of prescriptions, one with $\mathrm{BED}_{10}$ of 105.6 Gy and the other with $\mathrm{BED}_{10}$ of $105 \mathrm{~Gy}$, were compared, local control rates differed markedly despite only a slight difference in $\mathrm{BED}_{10}$ (Figure 2).

BED calculated by the linear quadratic formula with no correction was used in this study. The factor of overall radiation time was therefore not taken into account. During prolonged radiation delivery, sublethal damage repair takes place, leading to a decreased effect of radiation [20]. Actually, there was no local recurrence in patients with the shortest radiotherapy (105.6 Gy BED Bo $_{10}$ in this study, although 6 patients treated with $105.6 \mathrm{~Gy}$ $\mathrm{BED}_{10}$ had a T2 tumor.

Many clinicians often use the linear-quadratic (LQ) model and BED to estimate the effects of various radiation schedules, but it has been suggested that the LQ model is not applicable to stereotactic radiotherapy because of its high dose per fraction [20]. By contrast, Fowler et al. reported that the LQ model fitted the radiation response of epithelial tissues $<23$ Gy/fraction [16].
The best-fit model for tumor responses to stereotactic radiotherapy warrants further research.

Furthermore, we speculate that restriction of prescribed doses due to the vicinity of central structures and/or the radiation oncologist's discretion in consideration of factors including performance status affected the control rate, resulting in this difference (Figure 2).

Some reports have shown that local control rate with over 100 Gy $\mathrm{BED}_{10}$ was higher than that with less than 100 Gy $\mathrm{BED}_{10}$ [3], although a meta-analysis conducted by Zhang et al. showed no significant difference between $\mathrm{BED}_{10}<100$ Gy and $\mathrm{BED}_{10} \geq 100$ Gy [21]. Medium $\mathrm{BED}$ or medium to high BED were recommended by Zhang et al. In our study, 4 Gy/fraction $\times 15$ fractions $\left(84\right.$ Gy $\mathrm{BED}_{10}$ ) was suggested to be insufficient for treatment of stage I NSCLC, particular in patients with a T2 tumor.

Further studies are needed to clarify the optimal total dose and fractions and the risk factors for relapse and side effects. When lower BED has to be prescribed because of tumor size, location of the tumor,

Table 4 Reports of stereotactic radiotherapy for stage I non-small cell lung cancer

\begin{tabular}{llllllll}
\hline $\begin{array}{l}\text { First author } \\
\text { (Reference No.) }\end{array}$ & $\begin{array}{l}\text { Number of } \\
\text { patients }\end{array}$ & $\begin{array}{l}\text { Total dose } \\
\text { (Gy) }\end{array}$ & $\begin{array}{l}\text { Single dose } \\
\text { (Gy/fraction) }\end{array}$ & BED $_{\mathbf{1 0}}$ (Gy) & $\begin{array}{l}\text { Median follow up } \\
\text { (months) }\end{array}$ & $\begin{array}{l}\text { Local control } \\
\text { (\%)** }\end{array}$ & $\begin{array}{l}\text { 3-year overall } \\
\text { survival (\%) }\end{array}$ \\
\hline Timmerman [6] & 55 & 60 & 20 & 180 & 34.4 & 98.2 & 55.8 \\
Nagata [5]* & 42 & 48 & 12 & 105.6 & 30 & 97.8 & 83 \\
Fakiris [7] & 70 & 60,66 & 20,22 & $180-211.2$ & 50.2 & 94.3 & 42.7 \\
Onishi [3]* & 257 & $18-75$ & $4.4-35$ & $57.6-180$ & 38 & 88.7 & 56.8 \\
Our results* & 80 & $48-60$ & $4-12$ & $84-105.6$ & 30.4 & 92.5 & 89.9 \\
\hline
\end{tabular}

$\mathrm{BED}_{10}$ is biological effective dose (BED) calculated from prescribed dose.

* Dose is calculated at the iso-center.

**Local control $(\%)=$ (number of patients without local recurrence) $/$ (number of all patients) $\times 100$. 
and/or complications, use with chemotherapy can be considered [22].

\section{Minimum dose for PTV (\%)}

Wald $\chi^{2}$ test indicated that minimum dose for PTV (\%) was the strongest prognostic factor. We carried out the calculation of the local control, grouping by the minimum dose for PTV (\%) into 2 groups: the minimum dose for PTV $(\%) \geq 89.88 \%$ and $<89.88 \%$, and there was significant difference. Baumann et al. showed by univariate analysis that radiation dose calculated in equivalent doses in 2 Gy fractions (EQD2) at the periphery of the PTV had an impact on survival but not on local recurrence rate [15]. A trend toward smaller PTV margins for recurrent patients was also observed in their phase II trial [23]. Among multiple factors, BED at the PTV margin was found to be the only significant factor influencing local control by Wulf et al. [24].

BED at the PTV margin can decrease for many reasons. The internal margin is not constant in each fraction, and sufficient management of the respiration factor is therefore important for planning particularly in the lower lobe. Dosimetric problems also arise from the limited accuracy of dose calculation algorithms in treatment planning systems. Lax et al. reported that the pencil beam algorithm significantly overestimates the dose [25]. Radiation oncologists must have knowledge of the characteristics of the algorithm used in each institution. According to some reports, irradiation for large volumes of the lung can result in high-grade radiation pneumonitis [26]. Radiation pneumonitis is the most important adverse reaction in stereotactic radiotherapy for a lung tumor and its prevention is a crucial task because of its severity. For this reason, the leaf margin might be reduced at the discretion of the radiation oncologist when the target is large and/or the patient has respiratory complications.

Efforts to avoid unnecessary reduction of the margin should be made. Nowadays, we use the dose prescribed by $95 \%$ of the PTV (D95) for the stereotactic radiotherapy. We will conduct further research to determine an acceptable minimum dose (\%).

The continuing development of technologies including respiratory gated radiotherapy and real-time tumor tracking should enable further reduction of the margin for PTV. Therefore, further dose escalation for a larger tumor such as a T2 tumor may be possible, and severe side effects may be reduced since the amount of normal tissue irradiation will also be reduced with reduction of the margin for PTV.

\section{Conclusion}

$\mathrm{T}$ factor, $\mathrm{BED}_{10}$, and minimum dose for PTV (\%) influence the local control rate. Local control rates can be improved by securing the minimum dose for PTV when radiation oncologists produce a radiotherapy plan for stage I nonsmall cell lung cancer in stereotactic radiotherapy.

\section{Competing interests}

The authors declare that they have no competing interests.

\section{Authors' contributions}

$\mathrm{KJ}$ and MK conceived of the study. YS and MK participated in data collection. YS performed the statistical analysis and drafted the manuscript. KJ, MK, KT, and NK helped to draft the manuscript. KJ, TS, and HM critically reviewed the article. All authors read and approved the final manuscript.

\section{Author details}

'Department of Radiation Oncology, Tohoku University School of Medicine, Sendai, Japan. ${ }^{2}$ Research Center for Charged Particle Therapy, National Institute of Radiological Sciences, Chiba, Japan. ${ }^{3}$ Department of Therapeutic Radiology, Clinical Radiological Science, Course of Radiological Technology, Tohoku University School of Health Sciences, Sendai, Japan. ${ }^{4}$ Department of Radiation Oncology, Tohoku University School of Medicine, 1-1 Seiryo-chou, Aoba-ku, Sendai 980-8574, Japan.

Received: 13 July 2012 Accepted: 26 October 2012

Published: 31 October 2012

\section{References}

1. Buyyounouski MK, Balter $P$, Lewis B, et al: Stereotactic body radiotherapy for early-stage non-small-cell lung cancer: report of the ASTRO emerging technology committee. Int J Radiat Oncol Biol Phys 2010, 78:3-10.

2. Onimaru R, Fujino M, Yamazaki K, et al: Steep dose-response relationship for stage I non-small-cell lung cancer using hypofractionated high-dose irradiation by real-time tumor-tracking radiotherapy. Int J Radiat Oncol Biol Phys 2008, 70:374-381.

3. Onishi H, Shirato H, Nagata Y, et al: Hypofractionated Stereotactic Radiotherapy (HypoFXSRT) for Stage I Non-small cell lung cancer: Updated results of 257 patients in a Japanese multi-institutional study. J Thorac Oncol 2007, 2:S94-S100.

4. Asamura H, Goya T, Koshiishi Y, et al: A Japanese Lung Cancer Registry Study: Prognosis of 13010 Resected Lung Cancers. J Thorac Oncol 2007, 3:46-52.

5. Willard AF, Jerri LP, Herman RM: Ten-year survey of lung cancer treatment and survival in hospitals in the United States. A national cancer data base report. Cancer 1999, 86(9):1867-1876.

6. National Lung Screening Trial Research Team, Aberle DR, Adams AM, Berq CD, Black WC, Clapp JD, Fagerstrom RM, Gareen IF, Gatsonis C, Marcus PM, Sicks JD: Reduced lung-cancer mortality with low-dose computed tomographic screening. N Engl J Med 2011, 365(5):395-409.

7. Nagata Y, Takayama K, Matsuo Y, Norihisa Y, Mizowaki T, Sakamoto T, Sakamoto M, Mitsumori M, Shibuya K, Araki N, Yano S, Hiraoka M: Clinical outcomes of a phase I/II study of 48 Gy of stereotactic body radiotherapy in 4 fractions for primary lung cancer using a stereotactic body frame. Int J Radiat Oncol Biol Phys 2005, 63(5):1427-1431.

8. Timmerman R, Paulus R, Galvin J, Michalski J, Straube W, Bradley J, Fakiris A, Bezjak A, Videtic G, Johnstone D, Fowler J, Gore E, Choy H: Stereotactic body radiation therapy for inoperable early stage lung cancer. JAMA 2010, 303(11):1070-1076.

9. Fakiris AJ, McGarry RC, Yiannoutsos CT, Papiez L, Williams M, Henderson MA, Timmerman R: Stereotactic body radiation therapy for early-stage non-small-cell lung carcinoma: four-year results of a prospective phase II study. Int J Radiat Oncol Biol Phys 2009, 75(3):677-682.

10. Koto M, Takai Y, Ogawa Y, Matsushita H, Takeda K, Takahashi C, Britton KR, Jingu K, Takai K, Mitsuya M, Nemoto K, Yamada S: A phase II study on stereotactic body radiotherapy for stage I non-small cell lung cancer. Raditother Oncol 2007, 85(3):429-434.

11. Shiono S, Abiko M, Sato T: Positron emission tomography/computed tomography and lymphovascular invasion predict recurrence in stage I lung cancers. J Thorac Oncol 2010, 6:43-47.

12. Takeda A, Kunieda E, Shigematsu N, Hossain DM, Kawase T, Ohashi T, Fukada J, Kawaguchi O, Uematsu M, Takeda T, Takemasa K, Takahashi T, Kubo A: Small lung tumors: long-scan -time CT for planning of 
hypofractionated stereotactic radiation therapy-initial findings. Radiology 2005, 237:295-300

13. Matsuo Y, Shibuya K, Nagata Y, Takayama K, Norihisa Y, Mizowaki T, Narabayashi M, Sakanaka K, Hiraoka M: Prognostic factors in stereotactic body radiotherapy for non-small -cell lung cancer. Int J Radiat Oncol Biol Phys 2011, 79:1104-1111

14. Koike T, Tsuchiya R, Goya T, Sohara Y, Miyaoka E: Prognostic factors in 3315 Completely Resected Cases of Clinical Stage I Non-small Cell Lung Cancer in Japan. J Thorac Oncol 2007, 2:408-413.

15. Baumann P, Nyman J, Lax I, Friesland S, Hoyer M, Ericsson SR, Johansson KA, Ekberg L, Morhed E, Paludan M, Wittgren L, Blomgren H, Lewensohn R: Factors important for efficacy of stereotactic body radiotherapy of medically inoperable stage I lung cancer. A retrospective analysis of patients treated in the Nordic countries. Acta Oncol 2006, 45:787-795.

16. Fowler JF, Tomé WA, Fenwick JD, Mehta MP: A challenge to traditional radiation oncology. Int J Radiat Oncol Biol Phys 2004, 60:1241-1256.

17. Brunner TB, Kunz-Schughart LA, Grosse-Gehling P, Baumann M: Cancer Stem Cells as a Predictive Factor in Radiotherapy. Semin Radiat Oncol 2012, 22:151-174.

18. Matsuo Y, Shibuya K, Nagata Y, Norihisa Y, Narabayashi M, Sakanaka K, Ueki N, Mizowaki T, Hiraoka M: Preliminary Report of Late Recurrences, at 5 Years or more, after Stereotactic Body Radiation Therapy for Non-small Cell Lung Cancer. J Thorac Oncol 2012, 7:453-456.

19. Baba F, Shibamoto Y, Ogino H, Murata R, Sugie C, Iwata H, Otsuka S, Kosaki K, Nagai A, Murai T, Miyakawa A: Clinical outcomes of stereotactic body radiotherapy for stage I non-small cell lung cancer using different doses depending on tumor size. Radiat Oncol 2010, 5(81):1-7.

20. Shibamoto Y, Otsuka S, Iwata H, Sugie C, Ogino H, Tomita N: Radiobiological Evaluation of the Radiation Dose as Used in High-precision Radiotherapy: Effect of Prolonged Delivery Time and Applicability of the Linear-quadratic Model. J Radiat Res 2012, 53:1-9.

21. Zhang J, Yang F, Li B, Li H, Liu J, Huang W, Wang D, Yi Y, Wang J: Which is the optimal biological effective dose of stereotactic body radiotherapy for stage I non-small-cell lung cancer? A meta-analysis. Int J Radiat Oncol Biol Phys 2011, 81:e305-e316.

22. Chi A, Liao Z, Nguyen NP, Xu J, Stea B, Komaki R: Systemic review of the patterns of failure following stereotactic body radiation therapy in early-stage non-small-cell lung cancer: Clinical implications. Radiat Oncol 2010, 94:1-11.

23. Baumann P, Nyman J, Hoyer M, Wennberg B, Gagliardi G, Lax I, Drugge N, Ekberg L, Friesland S, Johansson KA, Lund JÅ, Morhed E, Nilsson K, Levin N, Paludan M, Sederholm C, Traberg A, Wittgren L, Lewensohn R: Outcome in a Prospective Phase II Trial of Medically Inoperable Stage I Non-Small Cell Lung Cancer Patients Treated With Stereotactic Body Radiotherapy. J Clin Oncol 2009, 27:3290-3296.

24. Wulf J, Baier K, Mueller G, Flentje MP: Dose-response in stereotactic irradiation of lung tumors. Radiat Oncol 2005, 77:83-87.

25. Lax I, Panettieri V, Berit W, et al: Dose-distributions in SBRT of lung tumors: Comparison between two different treatment planning algorithms and Monte-Carlo simulation including breathing motions. Acta Oncol 2006 45:978-988.

26. Yamashita H, Nakagawa K, Nakamura N, Duch MA, Näslund I, Baumann P, Gagliard G: Exceptionally high incidence of symptomatic grade 2-5 radiation pneumonitis after stereotactic radiation therapy for lung tumors. Radiat Oncol 2007, 2:1-11.

doi:10.1186/1748-717X-7-182

Cite this article as: Shirata et al:: Prognostic factors for local control of stage I non-small cell lung cancer in stereotactic radiotherapy: a retrospective analysis. Radiation Oncology 2012 7:182.

\section{Submit your next manuscript to BioMed Central and take full advantage of:}

- Convenient online submission

- Thorough peer review

- No space constraints or color figure charges

- Immediate publication on acceptance

- Inclusion in PubMed, CAS, Scopus and Google Scholar

- Research which is freely available for redistribution

Submit your manuscript at www.biomedcentral.com/submit
C Biomed Central 